\title{
Ito-Sadahiro numbers vs. Parry numbers
}

\author{
Z. Masáková, E. Pelantová
}

\begin{abstract}
We consider a positional numeration system with a negative base, as introduced by Ito and Sadahiro. In particular, we focus on the algebraic properties of negative bases $-\beta$ for which the corresponding dynamical system is sofic, which happens, according to Ito and Sadahiro, if and only if the $(-\beta)$-expansion of $-\frac{\beta}{\beta+1}$ is eventually periodic. We call such numbers $\beta$ Ito-Sadahiro numbers, and we compare their properties with those of Parry numbers, which occur in the same context for the Rényi positive base numeration system.
\end{abstract}

Keywords: numeration systems, negative base, Pisot number, Parry number.

\section{Introduction}

The expansion of a real number in the positional number system with base $\beta>1$, as defined by Rényi [12] is closely related to the transformation $T:[0,1) \mapsto[0,1)$, given by the prescription $T(x):=$ $\beta x-\lfloor\beta x\rfloor$. Every $x \in[0,1)$ is a sum of the infinite series

$$
\begin{gathered}
x=\sum_{i=1}^{\infty} \frac{x_{i}}{\beta^{i}}, \quad \text { where } x_{i}=\left\lfloor\beta T^{i-1}(x)\right\rfloor \\
\text { for } i=1,2,3, \ldots
\end{gathered}
$$

Directly from the definition of the transformation $T$ we can derive that the 'digits' $x_{i}$ take values in the set $\{0,1,2, \ldots,\lceil\beta\rceil-1\}$ for $i=1,2,3, \ldots$. The expression of $x$ in the form (1) is called the $\beta$-expansion of $x$. The number $x$ is thus represented by the infinite word $d_{\beta}(x)=x_{1} x_{2} x_{3} \ldots \in \mathcal{A}^{\mathbb{N}}$ over the alphabet $\mathcal{A}=\{0,1,2, \ldots,\lceil\beta\rceil-1\}$.

From the definition of the transformation $T$ we can derive another important property, namely that the ordering on real numbers is carried over to the ordering of $\beta$-expansions. In particular, we have for $x, y \in[0,1)$ that

$$
x \leq y \quad \Longleftrightarrow \quad d_{\beta}(x) \preceq d_{\beta}(y),
$$

where $\preceq$ is the lexicographical order on $\mathcal{A}^{\mathbb{N}}$, (ordering on the alphabet $\mathcal{A}$ is usual, $0<1<2<\ldots<$ $\lceil\beta\rceil-1)$.

In [11], Parry has provided a criterion which decides whether an infinite word in $\mathcal{A}^{\mathbb{N}}$ is or not a $\beta$ expansion of some real number $x$. The criterion is formulated using the so-called infinite expansion of 1 , denoted by $d_{\beta}^{*}(1)$, defined as a limit in the space $\mathcal{A}^{\mathbb{N}}$ equipped with the product topology, by

$$
d_{\beta}^{*}(1):=\lim _{\varepsilon \rightarrow 0+} d_{\beta}(1-\varepsilon) .
$$

According to Parry, the string $x_{1} x_{2} x_{3} \ldots \in \mathcal{A}^{\mathbb{N}}$ represents the $\beta$-expansion of a number $x \in[0,1)$ if and only if

$$
\begin{aligned}
& x_{i} x_{i+1} x_{i+2} \ldots \prec d_{\beta}^{*}(1) \\
& \text { for every } i=1,2,3, \ldots
\end{aligned}
$$

Condition (2) ensures that the set $\mathcal{D}_{\beta}=\left\{d_{\beta}(x) \mid\right.$ $x \in[0,1)\}$ is shift invariant, and so the closure of $\mathcal{D}_{\beta}$ in $\mathcal{A}^{\mathbb{N}}$, denoted by $S_{\beta}$, is a subshift of the full shift $\mathcal{A}^{\mathbb{N}}$.

The notion of $\beta$-expansion can naturally be extended to all non-negative real numbers: The expression of a positive real number $y$ in the form

$$
\begin{gathered}
y=y_{k} \beta^{k}+y_{k-1} \beta^{k-1}+y_{k-2} \beta^{k-2}+\ldots, \\
\text { where } k \in \mathbb{Z} \text { and } y_{k} y_{k-1} y_{k-2} \ldots \in \mathcal{D}_{\beta},
\end{gathered}
$$

is called the $\beta$-expansion of $y$.

Real numbers $y$ having in the $\beta$-expansion of $|y|$ vanishing digits $y_{i}$ for all $i<0$ are usually called $\beta$ integers, and the set of $\beta$-integers is denoted by $\mathbb{Z}_{\beta}$. The notion of $\beta$-integers was first considered in [3] as an aperiodic structure modeling non-crystallographic materials with long range order, called quasicrystals. Numbers $y$ with finitely many non-zero digits in the $\beta$-expansion of $|y|$ form the set denoted by $\operatorname{Fin}(\beta)$.

The choice of the base $\beta>1$ strongly influences the properties of $\beta$-expansions. It turns out that an important role among bases is played by such numbers $\beta$ for which $d_{\beta}^{*}(1)$ is eventually periodic. Parry himself called these bases beta-numbers; now these numbers are commonly called Parry numbers. We can demonstrate the exceptional properties of Parry numbers on two facts:

- The subshift $S_{\beta}$ is sofic if and only if $\beta$ is a Parry number [6]. 
- Distances between consecutive $\beta$-integers take finitely many values if and only if $\beta$ is a Parry number [15].

Recently, Ito and Sadahiro [5] suggested a study of positional numeration systems with a negative base $-\beta$, where $\beta>1$. The representation of real numbers in such a system is defined using the transformation $T:\left[l_{\beta}, r_{\beta}\right) \mapsto\left[l_{\beta}, r_{\beta}\right)$, where $l_{\beta}=-\frac{\beta}{\beta+1}$, $r_{\beta}=1+l_{\beta}=\frac{1}{1+\beta}$,

$$
T(x):=-\beta x-\left\lfloor-\beta x-l_{\beta}\right\rfloor .
$$

Every real $x \in I_{\beta}:=\left[l_{\beta}, r_{\beta}\right)$ can be written as

$$
x=\sum_{i=1}^{\infty} \frac{x_{i}}{(-\beta)^{i}},
$$

where $x_{i}=\left\lfloor-\beta T^{i-1}(x)-l_{\beta}\right\rfloor$ for $i=1,2,3, \ldots$

The above expression is called the $(-\beta)$ expansion of $x$. It can also be written as the infinite word $d_{-\beta}(x)=x_{1} x_{2} x_{3} \ldots$ We can easily show from (4) that the digits $x_{i}, i \geq 1$, take values in the set $\mathcal{A}=\{0,1,2, \ldots,|\beta|\}$. In this case, the ordering on the set of infinite words over the alphabet $\mathcal{A}$ which would correspond to the ordering of real numbers is the so-called alternate ordering: We say that $x_{1} x_{2} x_{3} \ldots \prec_{\text {alt }} y_{1} y_{2} y_{3} \ldots$ if for the minimal index $j$ such that $x_{j} \neq y_{j}$ it holds that $x_{j}(-1)^{j}<y_{j}(-1)^{j}$. In this notation, we can write for arbitrary $x, y \in I_{\beta}$ that

$$
x \leq y \quad \Longleftrightarrow \quad d_{-\beta}(x) \preceq_{\text {alt }} d_{-\beta}(y) .
$$

In their paper, Ito and Sadahiro have provided a criterion to decide whether an infinite word $\mathcal{A}^{\mathbb{N}}$ belongs to the set of $(-\beta)$-expansions, i.e. to the set $\mathcal{D}_{-\beta}=\left\{d_{-\beta}(x) \mid x \in I_{\beta}\right\}$. This time, the criterion is given in terms of two infinite words, namely

$$
d_{-\beta}\left(l_{\beta}\right) \quad \text { and } \quad d_{-\beta}^{*}\left(r_{\beta}\right):=\lim _{\varepsilon \rightarrow 0+} d_{-\beta}\left(r_{\beta}-\varepsilon\right) .
$$

These two infinite words have a close relation: If $d_{-\beta}\left(l_{\beta}\right)$ is purely periodic with odd period length, i.e. $d_{-\beta}\left(l_{\beta}\right)=\left(d_{1} d_{2} \ldots d_{2 k+1}\right)^{\omega}$, then we have $d_{-\beta}^{*}\left(r_{\beta}\right)=$ $\left(0 d_{1} d_{2} \ldots\left(d_{2 k+1}-1\right)\right)^{\omega}$. (As usual, the notation $w^{\omega}$ stands for infinite repetition of the string $w$.) In all other cases we have $d_{-\beta}^{*}\left(r_{\beta}\right)=0 d_{-\beta}\left(l_{\beta}\right)$.

Ito and Sadahiro have shown that an infinite word $x_{1} x_{2} x_{3} \ldots$ represents a $(-\beta)$-expansion of some $x \in\left[l_{\beta}, r_{\beta}\right)$ if and only if for every $i \geq 1$ it holds that

$$
d_{-\beta}\left(l_{\beta}\right) \preceq_{\text {alt }} x_{i} x_{i+1} x_{i+2} \ldots \prec_{\text {alt }} d_{-\beta}^{*}\left(r_{\beta}\right) .
$$

The above condition ensures that the set $\mathcal{D}_{-} \beta$ of infinite words representing $(-\beta)$-expansions is shift invariant. In [5] it is shown that the closure of $\mathcal{D}_{-\beta}$ defines a sofic system if and only if $d_{-\beta}\left(l_{\beta}\right)$ is eventually periodic.

By analogy with the definition of Parry numbers, we suggest that numbers $\beta>1$ such that $d_{-\beta}\left(l_{\beta}\right)$ is eventually periodic be called Ito-Sadahiro numbers. The relation of the set of Ito-Sadahiro numbers and the set of Parry numbers is not obvious. Bassino [2] has shown that quadratic numbers, as well as cubic numbers which are not totally real, are Parry if and only if they are Pisot. For the same class of numbers, we prove in [10] that $\beta$ is Ito-Sadahiro if and only if it is Pisot. This means that notions of Parry numbers and Ito-Sadahiro numbers on the mentioned type of irrationals do not differ. This would support the hypothesis stated in the first version of this paper, namely that the set of Parry numbers and the set of Ito-Sadahiro numbers coincide. However, during the refereeing process Liao and Steiner [9] found an example of a Parry number which is not an ItoSadahiro number, and vice-versa.

The main results of this paper are formulated as Theorems 4 and 7 . Theorem 4 gives a bound on the modulus of conjugates of Ito-Sadahiro numbers; Theorem 7 shows that periodicity of $(-\beta)$-expansion of all numbers in the field $\mathbb{Q}(\beta)$ requires $\beta$ to be a Pisot or Salem number. Statements which we prove, as well as results of other authors that we recall, demonstrate similarities between the behaviour of $\beta$-expansions and $(-\beta)$-expansions. We mention also phenomena in which the two essentially differ.

\section{Preliminaries}

Let us first recall some number theoretical notions. A complex number $\beta$ is called an algebraic number, if it is a root of a monic polynomial $x^{n}+a_{n-1} x^{n-1}+\ldots+$ $a_{1} x+a_{0}$, with rational coefficients $a_{0}, \ldots, a_{n-1} \in \mathbb{Q}$. A monic polynomial with rational coefficients and root $\beta$ of the minimal degree among all polynomials with the same properties is called the minimal polynomial of $\beta$, and its degree is called the degree of $\beta$. The roots of the minimal polynomial are algebraic conjugates.

If the minimal polynomial of $\beta$ has integer coefficients, $\beta$ is called an algebraic integer. An algebraic integer $\beta>1$ is called a Perron number, if all its conjugates are in modulus strictly smaller than $\beta$. An algebraic integer $\beta>1$ is called a Pisot number, if all its conjugates are in modulus strictly smaller than 1 . An algebraic integer $\beta>1$ is called a Salem number, if all its conjugates are in modulus smaller than or equal to 1 and $\beta$ is not a Pisot number.

If $\beta$ is an algebraic number of degree $n$, then the minimal subfield of the field of complex numbers containing $\beta$ is denoted by $\mathbb{Q}(\beta)$ and is of the form

$$
\mathbb{Q}(\beta)=\left\{c_{0}+c_{1} \beta+\ldots+c_{n-1} \beta^{n-1} \mid c_{i} \in \mathbb{Q}\right\} .
$$


If $\gamma$ is a conjugate of an algebraic number $\beta$, then the fields $\mathbb{Q}(\beta)$ and $\mathbb{Q}(\gamma)$ are isomorphic. The corresponding isomorphism is given by

$c_{0}+c_{1} \beta+\ldots+c_{n-1} \beta^{n-1} \mapsto c_{0}+c_{1} \gamma+\ldots+c_{n-1} \gamma^{n-1}$.

In particular, this means that $\beta$ is a root of some polynomial $f$ with rational coefficients if and only if $\gamma$ is a root of the same polynomial $f$.

\section{Ito-Sadahiro polynomial}

From now on, we shall consider for bases of the numeration system only Ito-Sadahiro numbers, i.e. numbers $\beta$ such that

$$
d_{-\beta}\left(l_{\beta}\right)=d_{1} \ldots d_{m}\left(d_{m+1} \ldots d_{m+p}\right)^{\omega} .
$$

Without loss of generality we shall assume that $m \geq$ $0, p \geq 1$ are minimal values so that $d_{-\beta}\left(l_{\beta}\right)$ can be written in the above form. Recall that $l_{\beta}=-\frac{\beta}{\beta+1}$. Therefore (7) can be rewritten as

$$
\begin{aligned}
& -\frac{\beta}{\beta+1}=\frac{d_{1}}{-\beta}+\ldots+\frac{d_{m}}{(-\beta)^{m}}+ \\
& \left(\frac{d_{m+1}}{(-\beta)^{m+1}}+\ldots+\frac{d_{m+p}}{(-\beta)^{m+p}}\right) \sum_{i=0}^{\infty} \frac{1}{(-\beta)^{p i}},
\end{aligned}
$$

and after arrangement

$$
\begin{aligned}
0= & \frac{-\beta}{-\beta-1}+\frac{d_{1}}{-\beta}+\ldots+\frac{d_{m}}{(-\beta)^{m}}+\frac{(-\beta)^{p}}{(-\beta)^{p}-1} . \\
& \left(\frac{d_{m+1}}{(-\beta)^{m+1}}+\ldots+\frac{d_{m+p}}{(-\beta)^{m+p}}\right) .
\end{aligned}
$$

Multiplying by $(-\beta)^{m}\left((-\beta)^{p}-1\right)$, we obtain the following lemma.

Lemma 1 Let $\beta$ be an Ito-Sadahiro number and let $d_{-\beta}\left(l_{\beta}\right)$ be of the form (7). Then $\beta$ is a root of the polynomial

$$
\begin{aligned}
P(x)= & (-x)^{m+1} \sum_{i=0}^{p-1}(-x)^{i}+\left((-x)^{p}-1\right) . \\
& \sum_{i=1}^{m} d_{i}(-x)^{m-i}+\sum_{i=m+1}^{m+p} d_{i}(-x)^{m+p-i} .
\end{aligned}
$$

Such a polynomial is called the Ito-Sadahiro polynomial of $\beta$.

Corollary 2 An Ito-Sadahiro number is an algebraic integer of degree smaller than or equal to $m+p$, where $m, p$ are given by (7).

It is useful to mention that the Ito-Sadahiro polynomial is not necessarily irreducible over $\mathbb{Q}$. As an example one can take the minimal Pisot number. For such $\beta$, we have $d_{-\beta}\left(l_{\beta}\right)=1001^{\omega}$, and thus the Ito-Sadahiro polynomial is equal to $P(x)=$ $x^{4}-x^{3}-x^{2}+1=(x-1)\left(x^{3}-x-1\right)$, where $x^{3}-x-1$ is the minimal polynomial of $\beta$.
Remark 3 Note that for $p=1$ and $d_{m+1}=0$, we have $d_{-\beta}\left(l_{\beta}\right)=d_{1} \ldots d_{m} 0^{\omega}$, and the Ito-Sadahiro polynomial of $\beta$ is of the form

$$
\begin{aligned}
P(x)= & (-x)^{m+1}+d_{1}(-x)^{m}+\left(d_{2}-d_{1}\right)(-x)^{m-1}+ \\
& \ldots+\left(d_{m}-d_{m-1}\right)(-x)-d_{m},
\end{aligned}
$$

and thus $\beta$ is an algebraic integer of degree at most $m+1$.

Theorem 4 Let $\beta$ be an Ito-Sadahiro number. All roots $\gamma, \gamma \neq \beta$, of the Ito-Sadahiro polynomial (in particular all conjugates of $\beta$ ) satisfy $|\gamma|<2$.

Proof. $\quad$ Since $\beta$ is a root of its Ito-Sadahiro polynomial $P$, there must exist a polynomial $Q$ such that $P(x)=(x-\beta) Q(x)$. Let us first determine $Q$ and show that it is a monic polynomial with coefficients in modulus not exceeding 1 . The coefficients $d_{i}$ in the polynomial $P$ in the form (8) are the digits of the $(-\beta)$-expansion of $l_{\beta}$, and thus, by (5), they satisfy $d_{i}=\left\lfloor-\beta T^{i-1}\left(l_{\beta}\right)-l_{\beta}\right\rfloor$. Relation (4) then implies $T^{i}\left(l_{\beta}\right)=-\beta T^{i-1}\left(l_{\beta}\right)-\left\lfloor-\beta T^{i-1}\left(l_{\beta}\right)-l_{\beta}\right\rfloor$, wherefrom we have

$$
d_{i}=-T^{i}\left(l_{\beta}\right)-\beta T^{i-1}\left(l_{\beta}\right) .
$$

For simplicity of notation in this proof, denote $T_{i}=$ $T^{i}\left(l_{\beta}\right)$, for $i=0,1, \ldots, m+p$. Substituting $d_{i}=$ $-T_{i}-\beta T_{i-1}$ into (8), we obtain

$$
\begin{aligned}
P(x)= & (-x)^{m+1} \sum_{i=0}^{p-1}(-x)^{i}+\left((-x)^{p}-1\right) \cdot \\
& \sum_{i=1}^{m}\left(-T_{i}-\beta T_{i-1}\right)(-x)^{m-i}+ \\
& \sum_{i=m+1}^{m+p}\left(-T_{i}-\beta T_{i-1}\right)(-x)^{m+p-i}= \\
& (-x)^{m+1} \sum_{i=0}^{p-1}(-x)^{i}+\left((-x)^{p}-1\right)(x-\beta) \\
& \sum_{i=2}^{m} T_{i-1}(-x)^{m-i}+ \\
& (x-\beta) \sum_{i=1}^{p} T_{m+i-1}(-x)^{p-i}- \\
& \left((-x)^{p}-1\right) \beta T_{0}(-x)^{m-1}+T_{m}-T_{m+p} .
\end{aligned}
$$

First realize that $T_{m}-T_{m+p}=0$, since $d_{-\beta}\left(l_{\beta}\right)$ is eventually periodic with a preperiod of length $m$ and a period of length $p$. As $T_{0}=T^{0}\left(l_{\beta}\right)=-\frac{\beta}{\beta+1}$, we can derive that

$$
\begin{aligned}
& (-x)^{m+1} \sum_{i=0}^{p-1}(-x)^{i}-\left((-x)^{p}-1\right) \beta T_{0}(-x)^{m-1}= \\
& (-x)^{m-1}(x-\beta)\left(x-T_{0}\right) \sum_{i=0}^{p-1}(-x)^{i} .
\end{aligned}
$$


Putting back to (10), we obtain that the desired polynomial $Q$ defined by $P(x)=(x-\beta) Q(x)$ is of the form

$$
\begin{aligned}
Q(x)= & (-x)^{m-1}\left(x-T_{0}\right) \sum_{i=0}^{p-1}(-x)^{i}+ \\
& \left((-x)^{p}-1\right) \sum_{i=2}^{m} T_{i-1}(-x)^{m-i}+ \\
& \sum_{i=1}^{p} T_{m+i-1}(-x)^{p-i}
\end{aligned}
$$

which can be rewritten in another form, namely,

$$
\begin{aligned}
Q(x)= & -(-x)^{m+p-1}+ \\
& \sum_{i=m}^{m+p-2}\left(T_{m+p-1-i}-T_{0}-1\right)(-x)^{i}+ \\
& \sum_{i=0}^{m-1}\left(T_{m+p-1-i}-T_{m-1-i}\right)(-x)^{i} .
\end{aligned}
$$

Note that the coefficients at individual powers of $-x$ are of two types, namely

$$
T_{m+p-1-i}-T_{0}-1 \in[-1,0),
$$

and

$$
T_{m+p-1-i}-T_{m-1-i} \in(-1,1) .
$$

In order to complete the proof, realize that every root $\gamma, \gamma \neq \beta$, of the polynomial $P$ satisfies $Q(\gamma)=0$. We thus have

$$
\begin{aligned}
(-\gamma)^{m+p-1}= & \sum_{i=m}^{m+p-2}\left(T_{m+p-1-i}-T_{0}-1\right)(-\gamma)^{i}+ \\
& \sum_{i=0}^{m-1}\left(T_{m+p-1-i}-T_{m-1-i}\right)(-\gamma)^{i},
\end{aligned}
$$

and hence

$$
\begin{aligned}
|\gamma|^{m+p-1} \leq & \sum_{i=0}^{m+p-2}|\gamma|^{i}= \\
& \frac{|\gamma|^{m+p-1}-1}{|\gamma|-1}<\frac{|\gamma|^{m+p-1}}{|\gamma|-1} .
\end{aligned}
$$

From this, we easily derive that $|\gamma|<2$.

As a consequence, we can easily deduce the relation between Ito-Sadahiro numbers greater or equal to 2 and Perron numbers.

Corollary 5 Every Ito-Sadahiro number $\beta \geq 2$ is a Perron number.

In a recent preprint [9], it is shown that also ItoSadahiro numbers $\beta<2$ are Perron numbers.

\section{Periodic expansions in the Ito-Sadahiro system}

Representations of numbers in the numeration system with a negative base from the point of view of dynamical systems have been studied by Frougny and Lai [7]. They have shown the following statement.

Theorem 6 If $\beta$ is a Pisot number, then $d_{-\beta}(x)$ is eventually periodic for any $x \in I_{\beta} \cap \mathbb{Q}(\beta)$.

In particular, their result implies that every Pisot number is an Ito-Sadahiro number. Here, we show a 'reversed' statement.

Theorem 7 If any $x \in I_{\beta} \cap \mathbb{Q}(\beta)$ has eventually periodic $(-\beta)$-expansion, then $\beta$ is either a Pisot number or a Salem number.

Proof. First realize that since $l_{-\beta} \in \mathbb{Q}(\beta)$, by assumption, $d_{-\beta}\left(l_{\beta}\right)$ is eventually periodic, and thus $\beta$ is an Ito-Sadahiro number. Therefore, using Corollary $2, \beta$ is an algebraic integer. It remains to show that all conjugates of $\beta$ are in modulus smaller than or equal to 1 .

Consider a real number $x$ whose $(-\beta)$-expansion is of the form $d_{-\beta}(x)=x_{1} x_{2} x_{3} \ldots$ We now show that

$$
x_{1}=x_{2}=\ldots=x_{k-1}=0 \text { and } x_{k} \neq 0
$$

implies

$$
|x| \geq \frac{1}{\beta^{k}(\beta+1)} .
$$

In order to see this, we estimate the series

$|x|=\left|\frac{x_{k}}{(-\beta)^{k}}+\sum_{i=1}^{\infty} \frac{x_{k+i}}{(-\beta)^{k+i}}\right| \geq \frac{1}{\beta^{k}}-\frac{1}{\beta^{k}}\left|\sum_{i=1}^{\infty} \frac{x_{k+i}}{(-\beta)^{i}}\right|$.

Since the set $\mathcal{D}_{-\beta}$ of all $(-\beta)$-expansions is shift invariant, the sum $\sum_{i=1}^{\infty} \frac{x_{k+i}}{(-\beta)^{i}}$ is a $(-\beta)$-expansion of some $y \in I_{\beta}$. Therefore we can write

$$
|x| \geq \frac{1}{\beta^{k}}-\frac{1}{\beta^{k}}|y| \geq \frac{1}{\beta^{k}}-\frac{1}{\beta^{k}} \frac{\beta}{\beta+1}=\frac{1}{\beta^{k}(\beta+1)} .
$$

As $\beta>1$, there exists $L \in \mathbb{N}$ such that

$$
-\frac{\beta}{\beta+1}<\frac{1}{(-\beta)^{2 L+1}} \text {. }
$$

Let $M \in \mathbb{N}$ satisfy $M>2 L+1$. Choose a rational number $r$ such that

$$
\frac{1}{(-\beta)^{2 L+1}}<r<\frac{1}{(-\beta)^{2 L+1}}+\frac{1}{\beta^{M}(\beta+1)} .
$$

According to the auxiliary statement (12), the $(-\beta)$ expansion of $r$ must be of the form

$$
r=\frac{1}{(-\beta)^{2 L+1}}+\sum_{i=M+1}^{\infty} \frac{r_{i}}{(-\beta)^{i}} .
$$


As $r$ is rational, by assumption, the infinite word $r_{M+1} r_{M+2} \ldots$ is eventually periodic and by summing a geometric series, the sum $\sum_{i=M+1}^{\infty} \frac{r_{i}}{(-\beta)^{i}}$ can be rewritten as

$$
\sum_{i=M+1}^{\infty} \frac{r_{i}}{(-\beta)^{i}}=c_{0}+c_{1} \beta+\ldots+c_{n-1} \beta^{n-1} \in \mathbb{Q}(\beta),
$$

where $n$ is the degree of $\beta$.

In order to prove the theorem by contradiction, assume that a conjugate $\gamma \neq \beta$ is in modulus greater than 1. By application of the isomorphism between $\mathbb{Q}(\beta)$ and $\mathbb{Q}(\gamma)$, we get

$$
c_{0}+c_{1} \gamma+\ldots+c_{n-1} \gamma^{n-1}=\sum_{i=M+1}^{\infty} \frac{r_{i}}{(-\gamma)^{i}},
$$

and thus

$$
r=\frac{1}{(-\gamma)^{2 L+1}}+\sum_{i=M+1}^{\infty} \frac{r_{i}}{(-\gamma)^{i}}
$$

Subtracting (15) from (14), we obtain

$$
\begin{aligned}
0< & \left|\frac{1}{(-\beta)^{2 L+1}}-\frac{1}{(-\gamma)^{2 L+1}}\right| \leq \\
& \sum_{i=M+1}^{\infty} r_{i}\left|(-\beta)^{-i}-(-\gamma)^{-i}\right| \leq 2\lfloor\beta\rfloor \frac{\eta^{M+1}}{1-\eta}
\end{aligned}
$$

where $\eta=\max \left\{|\beta|^{-1},|\gamma|^{-1}\right\}<1$. Obviously, for any $M>2 L+1$, we can find a rational $r$ satisfying (13) and thus derive the inequality (16). However, the left-hand side of (16) is a fixed positive number, whereas the right-hand side decreases to zero with increasing $M$, which is a contradiction.

In order to stress the analogy of the Ito-Sadahiro numeration system with Rényi $\beta$-expansions of numbers, recall that already Schmidt in [13] has shown that for a Pisot number $\beta$, any $x \in[0,1) \cap \mathbb{Q}(\beta)$ has an eventually periodic $\beta$-expansion and also, conversely, that every $x \in[0,1) \cap \mathbb{Q}(\beta)$ having an eventually periodic $\beta$-expansion force $\beta$ is either a Pisot number or a Salem number. In fact, the proof of Theorem 6 given by Frougny and Lai, as well as our proof of Theorem 7 are using the ideas presented in [13].

A special case of numbers with periodic $(-\beta)$ expansion is given by those numbers $x$ for which the infinite word $d_{-\beta}(x)$ has suffix $0^{\omega}$. We then say that the expansion $d_{-\beta}(x)$ is finite. An example of such a number is $x=0$ with $(-\beta)$-expansion $d_{-\beta}(x)=0^{\omega}$. As is shown in [10], if $\beta<\frac{1}{2}(1+\sqrt{5})$, then $x=0$ is the only number with finite $(-\beta)$-expansion. This property of the Ito-Sadahiro numeration system has no analogue in Rényi $\beta$-expansions; for positive base, the set of finite $\beta$-expansions is always dense in $[0,1)$.
Just as in the numeration system with a positive base, we can extend the definition of $(-\beta)$-expansions of $x$ to all real numbers $x$, and define the notion of a $(-\beta)$-integer as a real number $y$ such that

$$
y=y_{k}(-\beta)^{k}+\ldots+y_{1}(-\beta)+y_{0},
$$

where $y_{k} \ldots y_{1} y_{0} 0^{\omega}$ is the $(-\beta)$-expansion of some number in $I_{\beta}$. The set of $(-\beta)$-integers is denoted by $\mathbb{Z}_{-\beta}$. With this notation, we can write the set of all numbers with finite $(-\beta)$-expansions as

$$
\operatorname{Fin}(-\beta)=\bigcup_{k=0}^{\infty} \frac{1}{(-\beta)^{k}} \mathbb{Z}_{-\beta}
$$

It is not surprising that the arithmetical properties of $\beta$-expansions and $(-\beta)$-expansions depend on the choice of the base $\beta$. It can be shown that both $\mathbb{Z}_{\beta}$ and $\mathbb{Z}_{-\beta}$ is closed under addition and multiplication if and only if $\beta \in \mathbb{N}$. On the other hand, $\operatorname{Fin}(\beta)$ and $\operatorname{Fin}(-\beta)$ can have a ring structure even if $\beta$ is not an integer. Frougny and Solomyak [8] have shown that if $\operatorname{Fin}(\beta)$ is a ring, then $\beta$ is a Pisot number. A similar result is given in [10] for a negative base: $\operatorname{Fin}(-\beta)$ being a ring implies that $\beta$ is either a Pisot number or a Salem number. In [10] we also prove the conjecture of Ito and Sadahiro that in the case of quadratic Pisot base $\beta$ the set $\operatorname{Fin}(-\beta)$ is a ring if and only if the conjugate of $\beta$ is negative.

\section{Comments and open questions}

- Every Pisot number is a Parry number and every Parry number is a Perron number, and neither of these statements can be reversed. The former is a consequence of the mentioned result of Schmidt, the latter statement follows for example from the fact that every Perron number has an associated canonical substitution $\varphi_{\beta}$, see [4]. The substitution is primitive, and its incidence matrix has $\beta$ as its eigenvalue. The fixed point of $\varphi_{\beta}$ is an infinite word which codes the sequence of distances between consecutive $\beta$-integers.

- For the negative base numeration system, we can derive from Theorem 6 that every Pisot number is an Ito-Sadahiro number. From Corollary 5 we know that an Ito-Sadahiro number $\beta \geq 2$ is a Perron number. Based on our investigation, we conjecture that for any Ito-Sadahiro number $\beta \geq \frac{1}{2}(1+\sqrt{5})$, the sequence of distances between consecutive $(-\beta)$-integers can be coded by a fixed point of a 'canonical' substitution which is primitive and its incidence matrix has $\beta^{2}$ for its dominant eigenvalue. Thus we expect that 
every Ito-Sadahiro number $\beta \geq \frac{1}{2}(1+\sqrt{5})$ is also a Perron number. In the case that $\beta<$ $\frac{1}{2}(1+\sqrt{5})$, we have $\mathbb{Z}_{-\beta}=\{0\}$ and so the situation is not at all obvious.

- In [14], Solomyak has explicitly described the set of conjugates of all Parry numbers. In particular, he has shown that this set is included in the complex disc of radius $\frac{1}{2}(1+\sqrt{5})$, and that this radius cannot be diminished. For his proof it was important that all conjugates of a Parry number are roots of a polynomial with real coefficients in the interval $[0,1)$. In the proof of Theorem 4 we show that conjugates of an Ito-Sadahiro number are roots of a polynomial (11) with coefficients in $[-1,1]$. From this, we derive that conjugates of Ito-Sadahiro numbers lie in the complex disc of radius $\leq 2$. We do not know whether this value can be diminished.

\section{Acknowledgement}

We acknowledge financial support from Czech Science Foundation grant 201/09/0584 and from grants MSM6840770039 and LC06002 of the Ministry of Education, Youth, and Sports of the Czech Republic.

\section{References}

[1] Ambrož, P., Dombek, D., Masáková, Z., Pelantová, E.: Numbers with integer expansion in the numeration system with negative base, preprint 2009, 13pp. http://arxiv.org/abs/0912.4597

[2] Bassino, F.: $\beta$-expansions for cubic Pisot numbers, 5th Latin American Theoretical INformatics Symposium (LATIN'02), 2286 LNCS. Cancun, Mexico. April, 2002. pp. 141-152. SpringerVerlag.

[3] Burdík, Č., Frougny, Ch., Gazeau, J. P., Krejcar, R.: Beta-Integers as Natural Counting Systems for Quasicrystals, J. Phys. A: Math. Gen. 31 (1998), 6449-6472.

[4] Fabre, S.: Substitutions et $\beta$-systèmes de numération, Theoret. Comput. Sci. 137 (1995), 219-236.
[5] Ito, S., Sadahiro, T.: (- $\beta$ )-expansions of real numbers, Integers 9 (2009), 239-259.

[6] Ito, S., Takahashi, Y.: Markov subshifts and realization of $\beta$-expansions, J. Math. Soc. Japan 26 (1974), 33-55.

[7] Frougny, Ch., Lai, A. C.: Negative bases and automata, Discr. Math. Theor. Comp. Sci. 13, No 1 (2011), 75-94.

[8] Frougny, Ch., Solomyak, B.: Finite $\beta$-expansions, Ergodic Theory Dynamical Systems 12 (1994), 713-723.

[9] Liao, L., Steiner, W.: Dynamical properties of the negative beta transformation, preprint 2011, 18pp. http://arxiv.org/abs/1101.2366

[10] Masáková, Z., Pelantová, E., Vávra, T.: Arithmetics in number systems with negative base, Theor. Comp. Sci. 412 (2011), 835-845.

[11] Parry, W.: On the $\beta$-expansions of real numbers, Acta Math. Acad. Sci. Hung. 11 (1960), 401-416.

[12] Rényi, A.: Representations for real numbers and their ergodic properties, Acta Math. Acad. Sci. Hung. 8 (1957), 477-493.

[13] Schmidt, K.: On periodic expansions of Pisot numbers and Salem numbers, Bull. London Math. Soc. 12 (1980), 269-278.

[14] Solomyak, B.: Conjugates of beta-numbers and the zero-free domain for a class of analytic functions, Proc. London Math. Soc. 68 (1994), 477-498.

[15] Thurston, W. P.: Groups, tilings, and finite state automata, AMS Colloquium Lecture Notes, American Mathematical Society, Boulder, 1989.

Zuzana Masáková

E-mail: zuzana.masakova@fjfi.cvut.cz

Edita Pelantová

E-mail: edita.pelantova@fjfi.cvut.cz

Department of Mathematics FNSPE

Czech Technical University in Prague

Trojanova 13, 12000 Praha 2, Czech Republic 